\title{
Géolinguistique
}

16 | 2016

Varia

\section{L'ALM : mort et résurrection d'un atlas linguistique de la culture méditerranéenne}

ALM: Death and Resurrection of a Linguistic Atlas of Mediterranean Culture

\section{Giovanni Ruffino et Tullio Telmon}

\section{QpenEdition}

\section{Journals}

\section{Édition électronique}

URL : http://journals.openedition.org/geolinguistique/452

DOI : $10.4000 /$ geolinguistique.452

ISSN : 2650-8176

\section{Éditeur}

UGA Éditions/Université Grenoble Alpes

\section{Édition imprimée}

Date de publication : 1 décembre 2016

Pagination : 21-39

ISBN : 978-2-84310-342-1

ISSN : 0761-9081

\section{Référence électronique}

Giovanni Ruffino et Tullio Telmon, «L'ALM : mort et résurrection d'un atlas linguistique de la culture méditerranéenne », Géolinguistique [En ligne], 16 | 2016, mis en ligne le 15 février 2019, consulté le 30 octobre 2020. URL : http://journals.openedition.org/geolinguistique/452 ; DOI : https://doi.org/ 10.4000/geolinguistique.452 


\title{
L'ALM : mort et résurrection d'un atlas linguistique de la culture méditerranéenne ${ }^{1}$
}

\author{
Giovanni Ruffino \\ Università di Palermo \\ Tullio Telmon \\ Università di Torino
}

\section{Résumé}

Conçu en 1937 par le romaniste croate Mirko Deanović et dirigé par luimême et l'italien Gianfranco Folena, l'Atlas linguistique de la Méditerranée a été réalisé à partir de 165 enquêtes et d'un questionnaire d'environ 850 items concernant la culture marine et maritime. Entre 1956 et 1972 il fût soumis par 31 enquêteurs à des témoins de tous les pays riverains de la Méditerranée. Tous les matériaux dialectaux avaient été déposés près de la Fondazione Giorgio Cini de Venise, qui fût également le commanditaire principal de la recherche. En 1972, un jeune chercheur turinois, Gaetano Berruto, fût chargé de la rédaction de ces matériaux, mais l'entreprise s'arrêta lorsque le rédacteur passa progressivement à d'autres obligations professionnelles et il ne fût plus remplacé. Un vrai trésor de connaissances linguistiques et ethnologiques est resté inexploité pendant cinquante ans. Finalement, en 2015 un groupe de géolinguistes de plusieurs universités italiennes a dressé un programme pour rafraîchir ces matériaux, les ranger, former une commission scientifique afin d'en vérifier les systèmes d'écriture phonétique et les rendre publiables.

1. Une version plus abrégée de ce texte a été présentée au IV Congrès international de dialectologie et de sociolinguistique organisé les 7, 8 et 9 septembre 2016 à l'université Paris-Sorbonne. 


\title{
Mots-clés
}

Atlas plurilingue, lexique marin, culture méditerranéenne.

\begin{abstract}
Conceived in 1937 by Croatian Romanist Mirko Deanović and directed by Deanović himself and the Italian scholar Gianfranco Folena, between 1956 and 1972 the Linguistic Atlas of the Mediterranean carried out 165 surveys by means of a questionnaire made up of about 850 items on sea and maritime culture. The surveys involved 31 researchers who interviewed people from all the countries on the coasts of the Mediterranean Sea. All the dialectal data were then sent to and filed at the Fondazione Giorgio Cini in Venice which was the first to commission the survey. In 1972, Gaetano Berruto, a young researcher form Turin, was entrusted with the editing of such data, but the study soon stopped since Berruto began to be engaged in different other work duties and was never replaced. A real ethnological and linguistic treasure remained untapped and unexplored for fifty years; finally in 2015 a team of geo-linguists coming from several Italian universities announced a programme to refresh the data, arrange them and check their phonetic notation system as preliminary steps to publish them.
\end{abstract}

\section{Keywords}

Polylingual atlas, sea lexicon, culture of mediterranean sea.

\section{Avant-propos}

Conçu en 1937 par le romaniste croate Mirko Deanović et dirigé par luimême et l'italien Gianfranco Folena, l'Atlas linguistique de la Méditerranée est le fruit de 165 enquêtes reposant sur un questionnaire de 810 items concernant la culture marine et maritime. Entre 1956 et 1972 il fût soumis à des témoins de tous les pays riverains de la Méditerranée, jusqu'à la mer Noire et à la mer d'Azov par 31 enquêteurs. Tous les matériaux dialectaux, remontants à trois familles linguistiques (indo-européenne, ouraloaltaïque, hamito-sémitiques) et (à l'époque) à 21 États, avaient été déposés auprès de la Fondazione Giorgio Cini de Venise, qui fût également le commanditaire principal de la recherche. En 1969, un jeune chercheur turinois, Gaetano Berruto, fût chargé de la rédaction de ces matériaux, mais l'entreprise s'arrêta lorsque, quelques années plus tard, le rédacteur passa progressivement à d'autres obligations professionnelles et il ne fût pas remplacé. 
La proposition de récupérer les matériaux de l'Atlas linguistique de la Méditerranée a été avancée au nom du Centro di Studi Filologici e Linguistici Siciliani, à l'occasion du $2^{\mathrm{e}}$ Colloque international sur l'étymologie et la géolinguistique romanes «Mare loquens», qui avait eu lieu à Zadar du 26 au 28 septembre 2013 (Ruffino, 2016). À l'occasion de la table ronde de clôture du XXI ${ }^{\mathrm{e}}$ Congrès international de linguistique et philologie romanes, dédiée à «La Méditerranée romane et la Sicile», en septembre 1995, les participants avaient exprimé: «[...] il rammarico che un'opera così importante per questo nostro straordinario angolo di mondo, sia tuttora e forse inesorabilmente incompiuta ${ }^{2} »$ (Ruffino, 1998: 619).

C'est donc le souvenir encore très frais de ce dernier congrès palermitain, mais aussi la conscience de l'actualité persistante des courants culturels et linguistiques qui continuent à traverser la Méditerranée, qui ont permis dernièrement d'entamer une profitable discussion entre spécialistes de différents domaines de la linguistique, intéressés à rendre exploitables les enquêtes de l'ALM. Le débat concerne les méthodes et les instruments les plus convenables pour mener à bien un projet de représentation diversifiée et modulaire des données, de façon telle à en permettre une jouissance aisée de la part de la communauté scientifique internationale ${ }^{3}$.

Un véritable trésor de connaissances linguistiques et ethnologiques est resté inexploité pendant cinquante ans; finalement, en 2015, un groupe de géolinguistes de plusieurs universités italiennes a dressé un programme pour rafraîchir ces matériaux, les ranger, former une commission scientifique pour en vérifier les systèmes d'écriture phonétique et les rendre publiables.

Sur l'élan d'un premier comité provisoire, composé par Giovanni Ruffino, Francesco Bruni, Michele Cortelazzo, Franco Crevatin, Carla Marcato et Tullio Telmon, en septembre 2015 s'est constitué un «Comité promoteur pour l'édition de l'ALM», dans le but, quelque peu hardi et audacieux, de conduire à son accomplissement l'œuvre amorcée par Deanović et Folena, dont les considérations qui ouvraient, il y a plus d'un demi-siècle, le pre-

2. «[...] le regret qu'une œuvre aussi importante pour notre petit coin de Monde, soit pour l'instant et peut être à jamais inachevée».

3. Nous faisons remarquer que les uniques matériaux $A L M$ publiés jusqu'ici (en plus du Saggio de 1971) concernent les enquêtes de cinq points siciliens: Mazara del Vallo, Porticello, Acitrezza, Lipari et Pantelleria (Ruffino, 1977). Le programme d'exploration du lexique de la mer en Sicile, entamé en 2010 (Ruffino \& D'Avenia, 2010) à l'intérieur de l'ALS (Atlante linguistico della Sicilia) et maintenant conclu avec l'enquête de Malta (Brincat \& D'Avenia, 2014), conseille de plus amples explorations et inspections. 
mier volume du Bollettino dell'ALM ${ }^{4}$, continuent à se démontrer d'une actualité surprenante. Aux spécialistes du comité provisoire, se sont ajoutés Gaetano Berruto (le premier rédacteur de l'ALM dans les années 19691972), Riccardo Contini, Patrizia Del Puente, Franco Fanciullo, Lorenzo Massobrio, Annalisa Nesi et Glauco Sanga.

Le parcours envisagé par le Comité prévoyait la signature d'un protocole d'entente avec la Fondazione Cini, la mise au point d'un projet de jouissance des matériaux de l'ALM, appuyé sur les pratiques informatiques les plus convenables, la création d'une plate-forme «internet», un plan d'appel de fonds locaux, une participation aux avis européens, pour le financement du projet dans son ensemble.

Du point de vue opérationnel, le Comité avait envisagé plusieurs phases, à partir d'une première inspection directe des matériaux pour en vérifier l'intégrité, pour arriver à la formation d'un Comité scientifique international, avec la tâche de superviser la digitalisation des 165 cahiers d'enquête originaux, ainsi distribués, du détroit de Gibraltar à la mer d'Azov: 1 point d'enquête au Portugal; 20 points en Espagne; 17 en France (y compris la Corse); 42 en Italie; 8 points sur la côte orientale de l'Adriatique (ancienne Yougoslavie); 3 points en Albanie; 23 en Grèce; 2 en Bulgarie; 3 en Roumanie; 12 dans l'ancienne URSS ; 8 en Turquie; 1 point à Chypre; 1 en Syrie; 2 au Liban, ainsi qu'en Israël; 3 points en Égypte; 4 en Lybie; 1 à Malte; 4 en Tunisie, ainsi qu'en Algérie et au Maroc.

La visite d'inspection dans les locaux de l'île de San Giorgio Maggiore, où la Fondazione Cini avait conservé toute la documentation de l'atlas, conduite dans les premiers jours de février 2016, a permis de retrouver et ranger les cahiers de toutes les enquêtes, ainsi que la correspondance entre les responsables et les enquêteurs pendant les travaux des enquêtes. Par la suite, durant une période malheureusement trop courte, Gaetano Berruto avait entamé son minutieux travail de rédaction, à partir de la révision de la transcription phonétique, dont les nombreux enquêteurs, appartenant à des traditions scientifiques souvent très différentes, avaient fini par multiplier les signes dans le souci de noter les nuances sonores les plus fines. G. Berruto avait également commencé à dresser 150 listes contenant les réponses de tous les points pour une même notion du questionnaire, en prévision de la réalisation des cartes respectives.

4. «Milieu géographique et écologique unitaire, la Méditerranée nous apparaît, depuis la plus haute Antiquité, vers laquelle la linguistique elle-même, avec l'ethnologie et l'archéologie, nous a habitué à tourner les yeux, un lieu prédestiné pour la rencontre de civilisations et de langues différentes, un trait d'union essentiel dans l'histoire du monde.» (Deanović \& Folena, 1959: 7) 
Compte tenu des nouvelles perspectives que la technologie informatique permet aujourd'hui, le Comité provisoire a décidé tout d'abord de procéder à une reproduction électronique des réponses contenues dans les cahiers. Ce travail est en train de se faire avec l'espoir qu'il sera très vite achevé.

La révision et la simplification des signes de transcription, facilitées par le travail déjà fait par G. Berruto, se fera donc sur les matériaux digitalisés, de façon à rendre plus aisée une probable translittération automatique du système de transcription phonétique originel (remontant à la tradition dialectologique Ascoli - Merlo, trop centrée sur le système italo-roman et quelque peu périmée) à un système plus actuel et répandu, comme pourrait être celui de l'API.

Mais ce sera au Comité scientifique international de prendre des décisions définitives sur cette matière: il devra de toute façon tenir compte de la nature éminemment lexicale, culturelle, ethnologique de l'entreprise et, par conséquent, du fait que les futurs utilisateurs de l'atlas se pencheront de préférence sur la recherche des convergences ou des différences motivationnelles (un facteur qui justifie à juste titre la démarche interlinguale de l'atlas) plutôt que sur des développements phonétiques qui ne peuvent à aucun titre être comparés d'une famille linguistique à l'autre.

Des contacts ont déjà été pris avec de nombreux collègues des pays méditerranéens, d'autres sont envisagés dans les mois prochains; un colloque/séminaire est déjà prévu à Palerme fin janvier 2017, organisée par le Centro di Studi Filologici e Linguistici Siciliani, afin d'installer le Comité scientifique international et pour prendre les décisions les plus urgentes vis-à-vis des problèmes de méthode, de ligne de conduite, et surtout de récolte de données.

\section{Quelques exemples}

\subsection{Ce que disent les vents du sud}

À l'occasion de notre présentation, lors du colloque annuel de Sappada en juillet 2015, nous avions employé les matériaux des atlas nationaux italiens (AIS et ALI) concernant les dénominations du «vent du Midi» (<(vento di) mezzogiorno > sur les côtes tyrrhéniennes; <ostro > sur les côtes adriatiques, sans compter les nombreux types mineurs, qui peuvent très souvent se superposer aux majoritaires). Nous avions conclu, entre autres, que probablement l'examen des données de la Méditerranée toute entière aurait pu confirmer ou démentir les hypothèses géolinguistiques et motivationnelles que nous avancions en l'occurrence (Ruffino \& Telmon, 2016: 107). La 
notion du «vent du Midi» fait justement partie des 25 notions cartographiées dans le Saggio de 1971 (Folena, Berruto \& Cortelazzo, 1971, carte $\mathrm{n}^{\circ} 66 \mathrm{Il}$ vento di $S$. $\left.(\text { Mezzogiorno })^{5}\right)$; il a été donc très séduisant de tenter une vérification de ces propos.

La lecture de la carte $n^{0} 66$ de l'ALM confirme, en effet, la première impression que nous avions eue en lisant les cartes des atlas nationaux, d'une sorte de double plan dénominatif: d'une part, celui des dénominations ponctuelles, locales, minimes et, d'autre part, celui des dénominations plus répandues (qui représentent très souvent le pendant ou le reflet dialectal des dénominations officielles dans les respectives langues nationales). Les nombreux cas de réponses multiples montrent que dans beaucoup de circonstances ces deux plans se superposent, donnant lieu à plusieurs synonymes. Étant donné que les situations sociolinguistiques «normales» de presque tous les répertoires peuvent se colloquer presque partout (excepté peut-être pour les dialectes arabes de la côte africaine) en condition de diglossie, nous pourrions avancer le mot «strato-synonymes» pour la plupart de ces cas de synonymie.

Un exemple: dans le point grec 114 Chanía (La Canée, île de Crète) les informateurs ont donné deux dénominations: [i no'tja] et [i 'ostrja] ${ }^{6}$ : si l'on ne réussit pas à trouver, dans les fichiers encore inexplorés de Venise, des notes d'enquête qui nous illuminent sur des emplois fonctionnels différenciés, il ne reste qu'à prendre acte que la deuxième forme, remontant au type le plus répandu le long de l'ensemble des côtes adriatiques et égéennes, pourrait être considérée la plus «haute», alors que la première, dont l'extension est limitée à l'Égée, devrait être la plus populaire (et probablement la plus ancienne).

Cela implique que les données de la carte $\mathrm{n}^{\circ} 66$ de l'ALM peuvent amener à deux lectures: la première visant à identifier les types lexicaux les plus répandus et à les mettre en évidence le long des côtes méditerranéennes, et une seconde lecture, distribuant les «strato-synonymes» mineurs en essayant d'y trouver les convergences motivationnelles. En ce qui concerne la première lecture, cinq lexotypes émergent. Si l'on excepte le type <notos, notià>, qui est toujours monorhémathique dans toutes ses 16 attestations, les lexotypes que l'on relève par cette lecture peuvent se présenter avec leur forme simple ou bien avec des suffixes altératifs, ou encore sous forme polyrhématique, c'est à dire déterminés (par des adjectifs, des appositions, des spécifications) ou bien comme déterminants eux-

5. Le vent du sud (Midi).

6. Nous avons retranscrit en API la notation phonétique. 
mêmes d'autres appellatifs, la plupart des cas sont constitués par des géonymes génériques ayant le sémantisme de «vent»: bjento, ven $(t)$, bènti, wèntu, vintê, rih, etc.

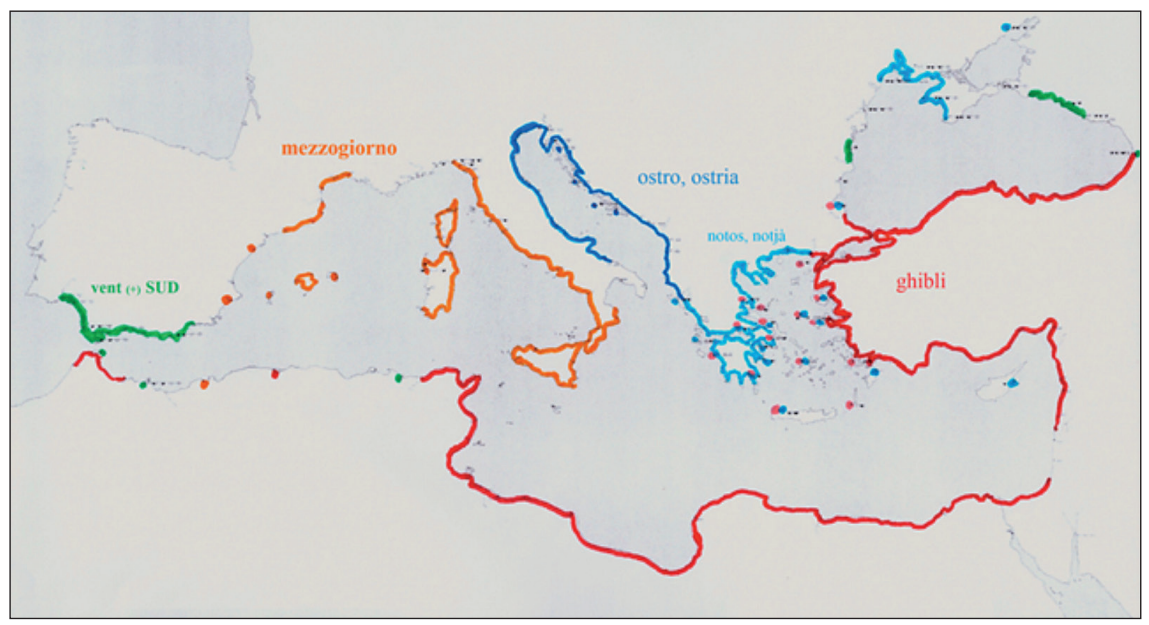

Carte $n^{\circ}$ 1. - Distribution des types lexicaux principaux sur les côtes de la Méditerranée, d'après la carte n ${ }^{\circ} 66$ de l'ALM.

En ce qui concerne les occurrences, le type le plus répandu est <ostro, ostria>, attesté dans 59 points d'enquête le long des côtes occidentales et orientales de l'Adriatique, sur les côtes et dans les îles de la Grèce ${ }^{7}$, sur les côtes bulgares et déjà soviétiques de la mer Noire, à Chypre.

7. La distribution des lexotypes dans les 23 points grecs mérite un approfondissement, on peut remarquer que:

1) tous les 23 points ont l'un ou l'autre ou les deux types;

2) en plus de <ostro, ostria $>$ et de <notos, notjà $>$, 2 points seulement présentent d'autres types;

3) compte tenu des réponses multiples, <ostro, ostria $>$ a 17 occurrences, $<$ notos, notjà> en a 15 ;

4) dans l'ensemble, 9 points ont les deux types, 8 ont seulement $<$ ostro, ostria $>, 6$ le seul <notos, notjà>;

5) des 7 points de la terre ferme, 4 ont les deux types, 2 seulement <ostro, ostria>, 1 le seul <notos, notjà>;

6) des 16 points insulaires, 5 ont les deux types, 6 ont seulement $<$ ostro, ostria>, 5 le seul <notos, notjà>;

7) des 8 îles plus étendues, 3 ont les deux types, 4 seulement <ostro, ostria>, 1 le seul <notos, notjà>;

8) des 8 îles plus petites, 2 ont les deux types, 2 seulement $<$ ostro, ostria $>, 4$ le seul $<$ notos, notjà $>$. 
Suit le type <mezzogiorno>, avec 41 occurrences, distribuées de la Catalogne à la côte du golfe du Lion (où il adjoint le caractéristique suffixe -ALE(M) : mjeSurnàl, mjetSurnàu, et similia) et puis, avec un hiatus significatif sur la côte provençale, en Corse, Sardaigne et Sicile, et sur les côtes tyrrhéniennes et ioniennes de l'Italie. Un des points algériens (161 Mersel-Kebir) atteste aussi une forme (vjent mjesujórno), dont les caractéristiques phonologiques et lexicales semblent évoquer des contacts avec le matelotage catalan, plutôt que des vestiges de la colonisation française.

Le troisième lexotype (27 occurrences) est <ghibli>, que nous pouvons relever au point 131 Batumi, sur la côte est - autrefois soviétique - de la mer Noire, et puis, avec très peu de solutions de continuité, sur les côtes turques de la mer Noire, de la mer de Marmara, de la Méditerranée, dans les États du Proche-Orient (à l'exception de deux points israéliens, qui connaissent le type équivalant drómit «méridional», déterminant de ruakh «vent»), sur les côtes africaines de la Méditerranée, de l'Égypte au Maroc.

Seul, ou précédé par l'appellatif "vent ${ }^{8}{ }$, le lexotype <sud> occupe sans solution, avec ses 18 occurrences, la côte d'Ayamonte (qui à vrai dire est déjà atlantique) jusqu'au point 10 Áquilas. En remontant vers le nord la côte ibérique de la Méditerranée, le dernier village espagnol, avant de passer en Catalogne et à son lexotype <mezzogiorno>, est le point 11 Santiago de la Ribera: comme il arrive très souvent dans les aires de transition, dans ce village le «vent du Midi» est dénommé avec un type (<scirocco $>$ ) qui n'est ni espagnol ni catalan, mais qu'on retrouve ici et là, au point 37 Bonifacio (colonie génoise en Corse), au point 42 Marina di Torre Grande (Sardaigne), au point 55 Maratea et 64 Taranto (Italie du Sud), et au point 92 dans l'île grecque de Kérkyra/Corfou. Le type <sud> se retrouve au point 162 Melilla, port franc de souveraineté espagnole sur la côte marocaine, et, sur la mer Noire, en deux points roumains et trois points de l'ancienne Union soviétique.

Le type <notos, notjà> a trois caractéristiques qui le distinguent des types que l'on a examinés jusqu'ici: d'abord, c'est le seul qui se présente, dans les 16 points où il est attesté, sous l'aspect toujours monorhématique, c'est-à-dire qu'il n'est jamais le déterminant ni le déterminé d'autres mots. En second lieu, il est restreint à la seule Grèce, en dehors de laquelle il est attesté seulement au point 115 Sozopol, une ville de la Bulgarie méridionale, non loin de la Thessalie, dont la tradition de liens avec la Grèce remonte

8. bjento au point 2 Ayamonte (Espagne); vïnt au point 118 Constanta (Roumanie); vån au point 158 Bona (Algérie); Bjénto au point 164 Ceuta (Maroc, mais de souveraineté espagnole). 
à sa fondation, autour de 600 avant Jésus-Christ, à cette époque les colonisateurs milésiens changèrent le nom de la mer Noire (le Pontus Eusinus des

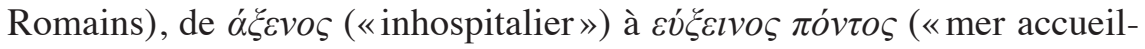
lante ») ${ }^{9}$. La troisième caractéristique est celle d'être l'unique, parmi les cinq lexotypes que l'on a retenus comme «majeurs», à ne pas se référer, pour la motivation, à la provenance méridionale. Si l'étymon proposé par la plupart des auteurs (vótoৎ «humide») est plausible, bien qu'en grec actuel ce mot ait pris aussi la signification de «sud», ce serait en effet le point cardinal à avoir reçu son nom du vent et non pas le vent du cadran de provenance, comme c'est le cas pour les quatre lexotypes discutés ci-dessus.

\subsection{Mots croisés}

Dans une de ses innombrables «réflexions méditerranéennes», Fernand Braudel parle de la Méditerranée comme «une image cohérente, comme un système où tout se mélange et se recompose en une unité originale» (1977).

Le Mare Nostrum des Romains peut donc être considéré comme un endroit ayant son unité intrinsèque ${ }^{10}$, dont le plurilinguisme représente, sur le plan linguistique, sa projection la plus ample: il devient ainsi un domaine où le linguiste même peut appliquer et vérifier cette condition de pluralité. Il faut dire tout d'abord que l'ALM, en tant qu'atlas concernant un territoire plurilingue très vaste, avec une référence spécifique au lexique de la mer, pose de délicats problèmes, étant donné que la diffusion des mots à travers les voies maritimes est bien différente de la diffusion qui se vérifie lorsqu'ils se diffusent le long des parcours terrestres. En outre, dans ce cadre, si sectoriel et étendu, il est également intéressant d'y rechercher non seulement des convergences mais aussi des divergences linguistiques, des phénomènes de fragmentation, d'atomisation même, à expliquer cas par cas.

9. Un écho de la création de cet euphémisme se trouve dans Ovide, Tristia, b. Iv, El. Iv. 56.

10. Très récemment, Calvet (2016) a introduit le terme séduisant de «continent liquide» pour désigner la Méditerranée dans son histoire linguistique et culturelle. 


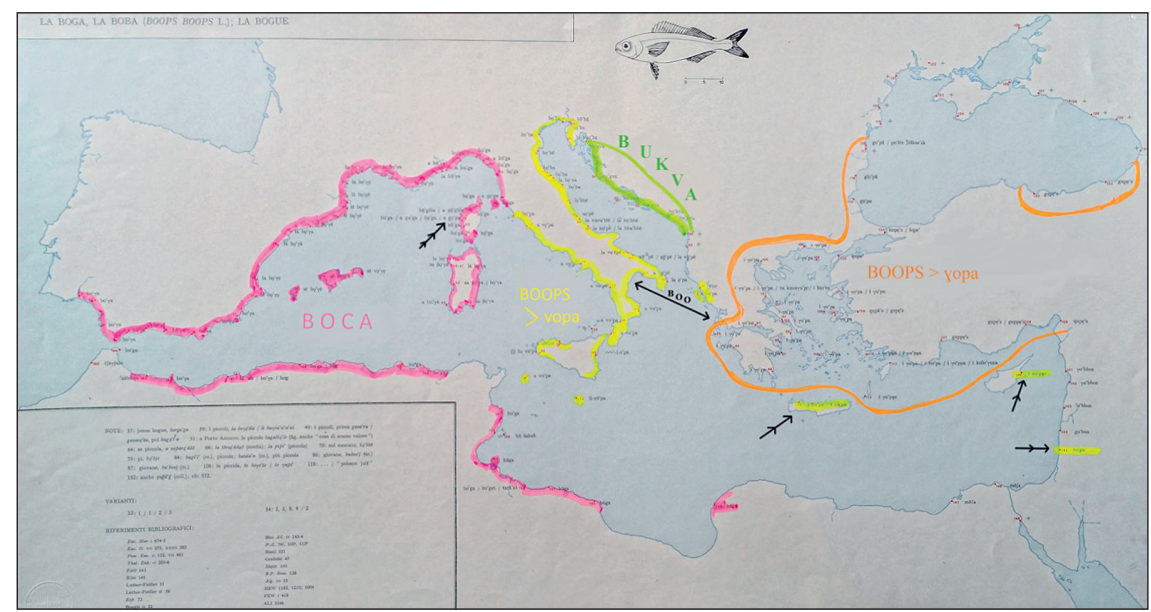

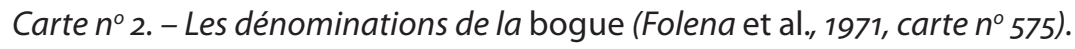

Juste pour citer quelques exemples, la position géographique souvent autonome de Chypre et Rhodes semble plutôt singulière par rapport à d'autres lieux grecs, comme pour les noms de la boga avec, dans les deux îles, sa variante adriatique et tyrrhénienne boba et sa variante grecque gopa qui appartient aussi à toute la Méditerranée orientale de la Turquie à la mer Noire.

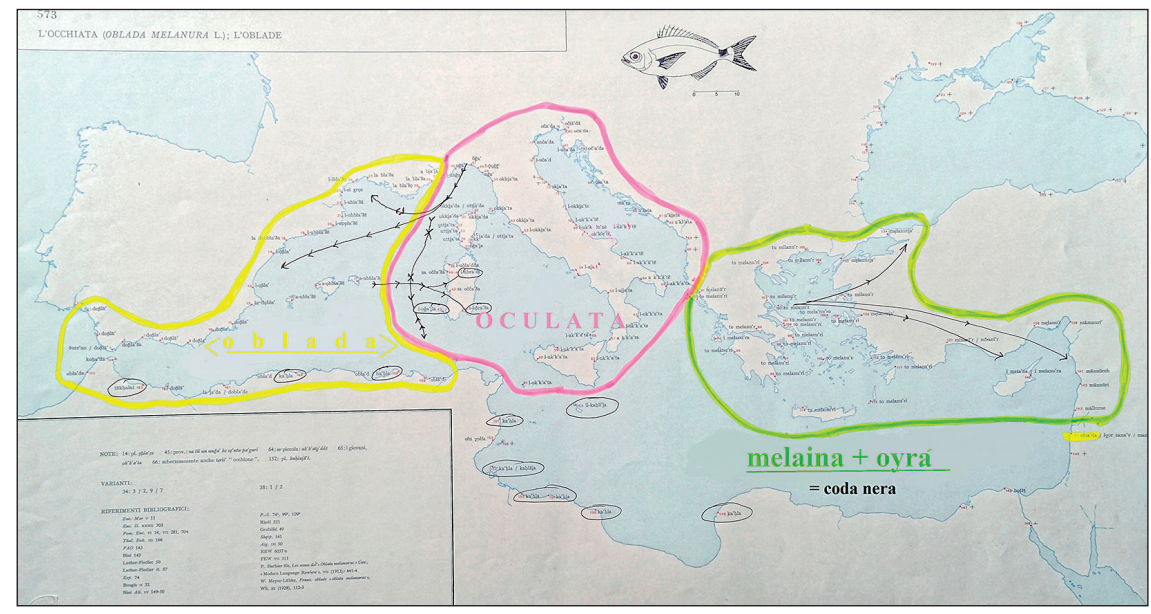

Carte $n^{\circ}$ 3. - Les dénominations de l'oblade (Folena et al., 1971, carte $n^{\circ}$ 573).

À ce propos, la carte d'épreuve concernant les noms de l'oblade (OBLADA MELANURA) est aussi révélatrice. Dans cette carte surgissent certains arrangements évidents du territoire, mais aussi la surprenante présence, limitée 
à Haifa, du lexotype <oblade>, typique des côtes franco-ibériennes, dans une zone où le type grecque <melanuri> est le plus diffusé.

Il s'agit, donc, d'une confirmation de ce que l'on disait auparavant sur la spécificité d'une géolinguistique de la mer et de la mer Méditerranée surtout, dont la diffusion plurilingue crée des agencements parfois aisément lisibles et d'autres fois, des parcours qui ne sont pas toujours faciles de retracer à nouveau.

Ces parcours, bien que tortueux, ont leur cohérence intime, comme celui du nom grec du poisson perke, la perche, par la suite latinisé, puis inséré dans la zone du Maghreb (berkăs) et, à partir de là, déplacé dans le territoire de langue romane (bircàcia, à Pantelleria et Lampedusa).

Au-delà de ces exemples si singuliers, l'observation et l'interprétation du lexique de la mer, du détroit de Gibraltar à la mer Noire, nous permettent d'individualiser une espèce de phénoménologie des dynamiques linguistiques et culturelles ayant traversé toute la mer. Un réseau d' «itinéraires lexicaux méditerranéens », aux routes unidirectionnelles (nord-sud ou sud-nord), bidirectionnelles (nord-sud et vice versa), et pluridirectionnelles. Un cadre de ce genre, au sein d'une circulation permanente de langues et cultures, souffre des variables dynamiques et des rapports de force que les langues de la Méditerranée ont exprimés à différentes époques.

Il suffit de penser aux rapports complexes entre latinité, grécité et dialectes berbères arabisés et entre ceux-ci et les variétés romanes, et aux réseaux réticulaires, moyennement larges, identifiables, par exemple dans le milieu ligurien/provençal, vénitien/balkanique, sicilien/maltais/maghrébin, dans le milieu de la Haute-Tyrrhénienne et de la Corse, dans celui qui inclut les îles Baléares, la Sardaigne, la Sicile avec des renvois liguriens/ provençaux/catalans ou encore, celui qui lie la zone grecque à celle turque/ balkanique jusqu'à la mer Noire.

Ces micro- et macro- convergences linguistiques impliquent une sorte de condition préliminaire consistant à accueillir et harmoniser, dans tout le vaste bassin, des éléments de différentes origines.

Ceci dit, une fois que l'on pourra consulter aisément les matériaux de l'Atlas linguistique de la Méditerranée, il sera possible d'avoir une évaluation géolinguistique la plus plausible, en introduisant ainsi de nouveaux éléments s'ajoutant aux déjà nombreuses sources documentaires. D'après quelques tentatives faites à l'aide des cahiers d'enquêtes consultés à Venise, on remarque des liens de «grands mots» approfondis et étudiés par d'influents intellectuels (il suffit de penser aux noms de certains bateaux ou du commerce maritime) et des liens de «petits mots» comme dans le domaine des noms des poissons. Dans les deux cas, toutefois, les doutes ne manquent pas et une réflexion renouvelée sur les sources de l'ALM pourrait 
bien sûr faire surgir de nouveaux éléments. Ainsi, d'après une observation des vastes sources de l'ALM, il peut y avoir d'intéressantes possibilités d'interprétation comme dans les cas de convergences de représentations sociales (motivationnelles, figuratives/sémantiques) que l'on pourrait définir «isosémantisme».

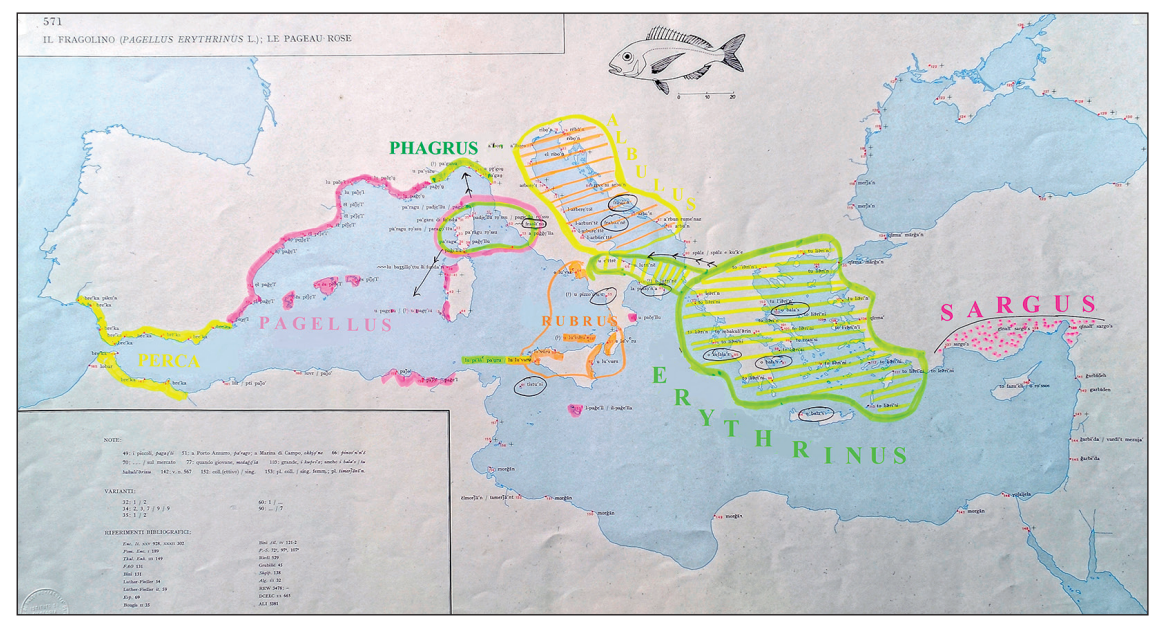

Carte $n^{\circ}$ 4. - Les dénominations du pageau rose (Saggio, 1971, carte $n^{\circ}$ 571).

On va citer le cas du PAGELLUS ERYTHRINUS qui, au-delà de nombreuses typologies lexicales, s'inscrit dans des zones figuratives/sémantiques qui se réfèrent à sa couleur rougeâtre: d'après les dérivés de RUBER/RUBRUS qui vont de la Campanie au Maroc à travers la Sicile, avec la variante RUBRONEM et ses variantes du Haut-Adriatique occidental et oriental, aux variantes de erythrínos, c'est-à-dire "poisson rouge», qui se trouvent en Grèce, en Albanie et le long des côtes des Pouilles, à l'arabe murğan, «corail» diffusé dans la zone du Maghreb jusqu'à la Bulgarie, vraisemblablement à travers la langue turque.

Un autre cas intéressant de convergences figuratives/sémantiques que nous avons récemment étudié concerne les nombreux noms de la méduse qui, avec les treize types lexicaux à individualiser à travers une longue série de variantes, se regroupent autour de différentes typologies motivationnelles parmi lesquelles on distingue le sens figuré des mots "génitales féminines» diffusé dans toute la Méditerranée avec des métaphores directes (les types conno, vulva, mona, pizda, mozza, etc.) ou avec des métaphores (p. ex. pota/potta) crées à partir d'un sens figuré précédent de vulva qui signifie «casserole», «poêle», «bol», largement représenté aussi dans les textes littéraires italiens du Moyen Âge. 
Il y a aussi la possibilité, donc, de construire des cartes typologiques/ motivationnelles où les dynamiques plurilingues se reconfigurent à l'intérieur d'une géolinguistique iconymique de la mer spéciale, qui rallie l'ALM avec les expériences, intervenues entretemps, des atlas interlinguaux de l'Europe (ALE) et de la Romania (ALiR).

\section{RÉFÉRENCES BIBLIOGRAPHIQUES}

AIS = JABERG Karl \& JuD Jakob, 1928-1940, Sprach- und Sachatlas Italiens und der Südschweiz, Zofingen.

ALI = MASSOBRIO Lorenzo (dir.), 1995-, Atlante linguistico italiano, 9 vol. publiés à ce jour, Rome, Istituto Poligrafico e Zecca dello Stato.

ALM = Deanović Mirko \& Folena Gianfranco, Atlante linguistico del Mediterraneo, Materiali dialettali inediti conservati presso la Fondazione Giorgio Cini, a Venezia.

B Aтtisti Carlo, 1962, «Sui grecismi dell'ittionimia latina. Accatti ed adattamenti di ittionimi greci nella tradizione latina», Bollettino dell'Atlante linguistico mediterraneo, $\mathrm{n}^{\circ} 4$, p. 37-52.

Berruto Gaetano, 1971-1973, «Problemi redazionali dell'ALM», Bollettino dell'Atlante linguistico mediterraneo, $\mathrm{n}^{\circ} 13-15$, p. 511-530.

Berruto Gaetano, 1976-1977, «Stato dei lavori dell'Atlante linguistico mediterraneo (ALM)», Bollettino dell'Atlante linguistico mediterraneo, $\mathrm{n}^{\circ}$ 18-19, p. 245-256.

BERRUTO Gaetano, 2001, «Il progetto dell'Atlante linguistico mediterraneo », dans U. Agnati (éd.), La fondazione Giorgio Cini. Cinquant'anni di storia, Milan, p. 145-149.

Braudel Fernand, 1977, La Méditerranée. L'espace et l'histoire, Paris, Arts et métiers graphiques.

Brincat Giuseppe \& D'Avenia Elena, 2014, L'inchiesta marinara a Malta, Palerme, Centro di studi filologici e linguistici siciliani.

CALVet Louis-Jean, 2016, La Méditerranée. Mer de nos langues, Paris, CNRS Éditions.

CCELC $=$ Corominas Joan \& Pascual Jose, 1980-1991, Diccionario critico etimológico castellano e hispánico, Madrid, Gredos.

Cortelazzo Manlio, 1989, Il Levante e il Mare, Pise, Pacini Editore.

CoRtelazzo Manlio, 1997, «Dal dizionario marinaresco elbano al dizionario degli ittionimi liguri», dans G. Marcato (éd.), I dialetti e il mare, Padoue, Unipress, p. 3-7.

Deanović Mirko \& Folena Gianfranco, 1959, «Prospettive dell'Atlante linguistico mediterraneo », Bollettino dell'Atlante linguistico mediterraneo, $\mathrm{n}^{\circ} 1$, p. 7-12.

Doria Mario, 1987, Grande dizionario del dialetto triestino storico etimologico fraseologico, Trieste, éd. Il Meridiano. 
Folena Gianfranco, Berruto Gaetano \& Cortelazzo Manlio, 1971, Saggio dell'Atlante linguistico mediterraneo, Florence, Olschki.

NALEC = DALbERA-STEFAnAggi Marie-Josée \& Miniconi Roger, 2008, Nouvel Atlas linguistique et ethnographique de la Corse, vol. 2, Ajaccio, éd. Alain Piazzola.

RID = LuRÀ Franco (dir.), 2013, Repertorio italiano-dialetti, Bellinzona, Centro di Dialettologia e di Etnografia.

Ruffino Giovanni, 1977, Il dialetto delle Pelagie e le inchieste dell'Atlante linguistico mediterraneo in Sicilia, Palerme, Centro di studi filologici e linguistici siciliani.

Ruffino Giovanni, 1998, Tavola rotonda su «Il Mediterraneo romanzo e la Sicilia», dans Atti del XXI Congresso internazionale di Linguistica e Filologia romanza (Palerme, 18-24 sept. 1995), vol. VI, Tübingen, Max Niemeyer.

Ruffino Giovanni, 2014, «Il mare nell'ALI», dans F. Cugno, L. Mantovani, M. Rivoira et M. S. Specchia (éds), Studi linguistici in onore di Lorenzo Massobrio, Turin, Istituto dell'Atlante linguistico italiano, p. 871-883.

RufFINo Giovanni, 2016, «Dall'Atlante linguistico mediterraneo all'Atlante linguistico della Sicilia: dinamiche areali e problemi storico-etimologici», dans N. Vuletić, X. A. Álvarez Pérez et J. E. Gargallo Gil (éds), Mari romanzi, mari del contatto: lessico e paremiologia, Zadar, Sveučilište u Zadru, p. 101-117.

Ruffino Giovanni \& D’Avenia Elena, 2010, Per un vocabolario-atlante della cultura marinara in Sicilia. Appunti e materiali, Palerme, Centro di studi filologici e linguistici siciliani.

Ruffino Giovanni \& Telmon Tullio, 2016, «L'Atlante linguistico del Mediterraneo. Quarant'anni dopo», dans G. Marcato (éd.), Il dialetto nel tempo e nella storia, Padoue, CLEUP, p. 95-116.

Simone Raffaele, 2010, Grande dizionario analogico della lingua italiana, Turin, UTET. 


\section{APPENDICES}

\section{Tableaux de synthèse des types lexicaux pour «le vent du Midi»}

Tableau 1. - Synthèse des types lexicaux les plus rependus pour le «vent du Midi».

1. Type <sud $>$ [Anglo-sax. anc. Sud (angl. mod. south)]

a) Espagne Points: 3 Palos de la Frontera, 4 San Fernando, 5 Algeciras, 6 Estepona, 7 Málaga, 8 Almuñécar, 9 Almería, 10 Áquilas.

b) Roumanie Points : 117 Eforie, 118 Constanta (Costanza).

c) URSS Points : 128 Tuapse, 129 Adler-Soci, 131 Batumi (Batum, Batoum).

d) Maroc Points : 162 Melilla.

1.1. Le même, mais comme déterminant de l'appellatif «vent» (<vent du sud>)
a) Espagne Points: 2 Ayamonte.
b) Roumanie Points : 118 Constanta (Costanza).
c) Algérie Points : 158 Annaba (Bona, Bône).
d) Maroc Points: 164 Ceuta.

2. Type <mezzogiorno> [Composé de lat. MEDIU(M) + DJURnU(M)]

a) Espagne/Catalogne Points : 12 Villajoyosa (La Vilajoiosa), 14 Peñíscola (Peníscola), 15 Ibiza (Eivissa), 16 Andraitx (Port d'Andratx), 17 Mahón (Maón), 19 Villanueva y Geltrú (Vilanova y la Geltrú), 20 Blanes, 21 La Escala.

b) France/Corse Points: 31 Calvi, 34 Bastia, 36 Propriano, 37 Bonifacio, 38 Porto-Vecchio.

c) Italie/Sardaigne Points: 39 Porto Torres, $41^{2}$ Alghero, 43 Carloforte, 44 Cagliari.

d) Italie Points : 47 Camogli, 48 Sestri Levante, 49 Monterosso al Mare, 50 Viareggio, 51 Portoferraio, 52 Porto S. Stefano, 53 Civitavecchia, 54 Portici.

2.1. Le même, mais comme déterminant de l'appellatif «vent» (<vent du mezzogiorno $>$ )

a) France/Corse Points: 33 Ajaccio.

b) Italie Points : 63 Crotone.

c) Algérie Points : 161 Mers-el-Kebir.

2.2. Avec le déterminant suffixale «-ale» (<mezzogiornale $>$ )

a) France Points: 23 Leucate, 24 Agde, 25 Grau-du-Roi.

2.3. Avec le déterminant suffixale alteratif «-accio» (<mezzogiornaccio $>$ )

a) Italie/Sicile Points: 59 Porticello.

2.4. Avec le déterminant suffixale altératif «-etto» (<mezzogiornetto $>$ )

a) Italie/Sicile Points : 59 Porticello.

2.5. Avec le déterminant adjectival «fresco » (<mezzogiorno fresco $>$ )

France/Corse Points : 32 Cargèse, 35 St.-Florent (S. Fiorenzo).

2.6. Avec le déterminant appositionel «libeccio» (<mezzogiorno libeccio $>$ )

a) Italie Points : 55 Maratea, 56 Pizzo, 57 Bagnara.

b) Italie/Sicile Points: 58 Lipari, 60 Mazzara del Vallo.

2.7. Avec le déterminant syntagmatique «di levante» (<mezzogiorno di levante $>$ )

a) Italie/Sicile Points: 62 Acitrezza. 
3. Type <ostro, ostria> [Lat. AUSTRU(M), gr. $o \sigma \tau \rho l \alpha$ ]

a) Italie Points: 68 Vasto, 69 Ortona, 70 Pescara, 72 Ancona (Ancône), 76 Porto Garibaldi, 77 Chioggia, 78 Grado, 80 Muggia.

b) Yougoslavie Points : 82 Rovinj (Rovigno), 83 Punat (Ponte), 84 Sali, 85 Komiza (Comisa), 86 Korcula (Cúrzola), 87 Cavtat (Ragusavecchia), 88 Muo (Mulla).

c) Albanie Points : 89 Shëngjin (S. Giovanni di Mèdua), 90 Durrës (Durazzo), 91 Sarandë (Santi Quaranta).

d) GrÈCE Points : 92 Kérkyra (Corfú, Corfou), 93 Argostólion (Argotoli), 94 Galaxídion, 95 Katákolon, 96 Monembasía (Malvasia, Malvoisie), 97 Hydra (Idra, Idhra), 98 Aígina (Egina, Égine), 100 Chalkís (Calcide, Chalcis), 103 Bólos (Volos), 104 Thessaloníke (Salonicco, Salonique), 105 Lágos (Porto Lago), 107 Mytiléne (Mitilene o Metelino, Mytilène ou Mételin), 108 Chíos (Chio o Scio), 109 Psará, 110 Páros, 111 Ródos (Rodi, Rhodes), 114 Chanía (La Canea, La Canée).

e) Bulgarie Points : 115 Sozopol.

f) URSS Points : 121 Odessa, 122 Kerson-Golaia, 123 Cernomorskoe (Ak-Mecet), 124 Sevastopol (Sebastopoli, Sébastopol), 126 Zdanov (Mariupol).

g) Chypre Points : 140 Ammóchostos (Famagosta, Famagouste).

3.1. Le même, avec la préposition de provenance «da» (<da ostro $\rangle$ )

a) Italie Points: 72 Ancona (Ancône), 73 Fano, 75 Cattòlica, 79 Santa Croce di Trieste (Sv. Kriz pri Trstu).

3.2. Le même, mais comme déterminant de l'appellatif «vent» (<vent di ostro $\rangle$ )

a) Italie Points : 67 Molfetta, 69 Ortona, $71 \mathrm{~S}$. Benedetto del Tronto, 74 Pesaro.

3.3. Avec le déterminant suffixale «-ale» (<ostrale $>$ )

a) Yougoslavie Points: 82 Rovinj (Rovigno), 84 Sali.

3.4. Avec le déterminant suffixale «-alata» $(<$ ostr(i)alata $>)$

a) Yougoslavie Points: 82 Rovinj (Rovigno), 88 Muo (Mulla).

3.5. Avec le déterminant suffixale altératif «-accio » (<ostraccio $\rangle)$

a) Yougoslavie Points: 84 Sali.

3.6. Avec le déterminant suffixale altératif «-ino» (<ostrino $\rangle)$

a) Yougoslavie Points: 84 Sali.

3.7. Avec le déterminant appellatif «scirocco» (<ostria scirocco $\rangle$ )

a) GRÈCE Points : 92 Kérkyra (Corfú, Corfou), 93 Argostólion (Argostoli).

3.8. Avec le déterminant appellatif «garbi(no)» (<ostrio garbis $>$ )

a) Bulgarie Points: 115 Sozopol.

4. Type <notos, notià> [gr. vótoऽ «humide»]

a) GrÈce Points : 94 Galaxídion, 95 Katákolon, 96 Monembasía (Malvasia, Malvoisie), 99 Rafína, 101 Néa Artáke, 102 Skíatos, 103 Bólos (Volos), 106 Samothráke (Samotracia, Samothtrace), 107 Mytiléne (Mitilene, Mytilène), 108 Chíos (Chio), 109 Psará, 110 Páros, 112 Koos (Coo), 113 Kásos (Caso), 114 Chanía (La Canea, La Canée).

b) Bulgarie Points: 115 Sozopol. 
5. Type <ghibli> [Ar. lybien qiblii «(vent) du sud)»]

a) URSS Points: 131 Batumi (Batum, Batoum).

b) Turquie Points : 132 Trabzon (Trebisonda, Trébizonde), 133 Samsun, 134 Istanbul (Costantinopoli, Constantinople), 135 Gelibolu (Gallipoli), 136 Izmir (Smirne, Smyrne), 137 Antalya (Adalia), 138 Mersin-Içel (Mersina), 139 Iskenderru (Alessandretta, Alexandrette).

c) ÉGYPTE Points : 147 El Iskandariiya (Alessandria, Alexandrie).

d) LiBye Points : 149 Benghazi (Bengasi), 150 Misraata (Misurata), 151 Tarabulus (Tripoli).

e) AlgÉRIE Points : 160 Cherchell.

f) MARoc Points : 163 Al-Hoceima, 164 Ceuta.

5.1. Le même, mais comme déterminant de l'appellatif «ri:h»(<ri:h ghibli>)

a) Syrie Points: 141 Arwaad (Ruad).

b) Liban Points: 142 Traablus (Tripoli), 143 Saidaa (Sidone, Sidon).

c) ÉGYPTE Points : 146 Dumyaat (Damietta, Damiette), 148 Es-Suweis (Suez).

d) Tunisie Points : 154 Houmt-Souk, 155 Sfax, 156 Qerqena (Kerkenna), 157 Mahdia.

e) Maroc Points: 165 El-Araich (Larache).

5.2. Le même, mais comme déterminant de l'appellatif «ádu» (<ádu d-aqåbli>)

a) LibYe Points: 152 Zuwaara (Zuara).

Tableau 2. - Synthèse des types lexicaux mineurs pour le «vent du Midi».

\section{Tipo <jug $>$}

1.1. Avec le déterminé «vjatər» (<juzen vjatar $>$ )

a) Bulgarie Points : 116 Varna.

b) URSS Points : 126 Zdanov (Mariupol').

\section{Type <scirocco>}

a) Espagne Points: 11 Santiago de la Ribera.

b) France/Corse Points : 37 Bonifacio

c) Italie Points : 42 Marina di Torre Grande, 55 Maratea, 64 Taranto (Tarente).

d) Grèce Points: 92 Kérkyra (Corfú, Corfou).

\section{Type <garbino>}

a) France Points: 22 Banyuls-sur-Mer.

b) Italie Points : 65 Otranto, 68 Vasto.

3.1. Le même, mais comme déterminant de l'appellatif «vento» (<vento a garbino $>$ )

a) Espagne/Catalogne Points: 13 Cullera.

\section{Type <libeccio>}

a) France Points : 23 Leucate, 29 Nice (Nizza), 30 Menton (Mentone).

\section{Type <ponente>}

a) Espagne/Catalogne Points : 18 Cala de l'Ametlla.

b) France Points : 28 St-Raphaël. 


\section{Type <dromít $>$}

6.1. Le même, mais comme déterminant de l'appellatif «rúah» (<rúah dromít>)

a) IsRaËL Points : 144 Heifa (Haifa), 145 Tel Aviv.

\section{Type <favonio>}

a) ITALIE Points : 66 Bari.

\section{Type $<$ da valle $>$}

8.1. Le même, mais comme déterminant de l'appellatif «vento» (<vento da valle>)

a) Espagne/Catalogne Points: 13 Cullera.

b) Maroc Points : 164 Ceuta.

\section{Type <mare $>$}

9.1. Le même, mais comme déterminant de l'appellatif «vento» (<vento di mare >)

a) Italie Points : $40^{1}$ Alghero.

\section{Type <la costa>}

10.1. Le même, mais comme déterminant de l'appellatif «bjento» (<bjento la kòhta>)
a) Maroc Points : 162 Melilla.

\section{Type <terra>}

11.1. Le même, mais comme déterminant de l'appellatif «vento» (<vento di terra>)

a) Italie/Sicile Points : 61 Pantelleria.

\section{Type <España $>$}

12.1. Le même, mais comme déterminant de l'appellatif «vento» (<vento di Spagna>)

a) France Points : 22 Banyuls-sur-Mer.

\section{Type <golfano>}

a) ItALIE Points : 66 Bari.

\section{Type <furlano>}
a) Yougoslavie Points: 82 Rovinj (Rovigno).

\section{Type <sole>}

15.1. Le même, mais comme déterminant de l'appellatif «vento» (<vento al sole $>$ )

a) Yougoslavie Points : 82 Rovinj (Rovigno).

\section{Type <korfos>}

a) GrÈce Points : 92 Kérkyra (Corfú, Corfou).

16.1. Avec le déterminant suffixal «-ana» (<korfana $>$ )

a) GrèCE Points : 92 Kérkyra (Corfú, Corfou).

\section{Type <Nisyros $>$}

17.1. Avec le déterminant suffixal «-iko» (<nisirico >)
a) Grece Points: $112 \mathrm{Koos}$ (Coo).

\section{Type <lodós>}
a) Bulgarie Points: 116 Varna.
b) Roumanie Points : 118 Constanta (Costanza).
c) URSS Points : 130 Sukhumi. 
19. Type <alb>

19.1. Le même, mais comme déterminant de l'appellatif «vento» (<vento alb>)

a) Roumanie Points : 118 Constanta (Costanza).

20. Type <polughiona, poludonka>

a) Roumanie Points : 119 Sfîntu Gheorghe (S. Giorgio).

b) URSS Points : 121 Odessa.

\section{Type <nizovka>}

a) URSS Points : 121 Odessa, 122 Kerson-Golaia, 125 Kerc, 127 Novorossijsk, 128 Tuapse.

22. Type <osman>

a) URSS Points: 131 Batumi (Batum, Batoum).

23. Type <kemalpashà>

a) URSS Points: 131 Batumi (Batum, Batoum).

24. Type <koerté>

a) Turquie Points : 132 Trabzon (Trebisonda, Trébizonde).

\section{Type <mazé $>$}

a) Turquie Points : 132 Trabzon (Trebisonda, Trébizonde).

\section{Type <aqiel>}

a) Turquie Points : 133 Samsun.

\section{Type <oksos cerós>}

a) Chypre Points: 140 Ammóchostos (Famagosta, Famagouste).

\section{Type <nofsináar $>$}

28.1. Le même, mais comme déterminant de l'appellatif «rïëh» (<riêh nofsináar>)

a) Malte Points : 153 Marsalforn (Marsalforno).

29. Type <bisa (?)>

a) Algérie Points : 159 Collo.

30. Type < glega $>$

a) Maroc Points : 164 Ceuta. 